\title{
Notas nomenclaturales, taxonómicas y corológicas sobre el género Cuphea (Lythraceae) en el Paraguay
}

\author{
REINILDA DURÉ(1) \& JULIÁN MOLERO(2)
}

\begin{abstract}
DURÉ, R. \& J. Molero (2000). Nomenclatural, taxonomic and chorologic notes on genus Cuphea (Lythraceae) in Paraguay. Collect. Bot. (Barcelona) 25: 225-243.

We present a nomenclatural study on genus Cuphea (Lythraceae) from Paraguay containing the correct name for 28 accepted species and their corresponding synonyms. We have revised the typification for most of the listed taxa. Subgenus Lytrocuphea Kochne, sect. Brachyandra Koehne and sect. Enatiocuphea Koehne are typified here. C. tuberosa Cham. \& Schltdl., C. inaequalifolia Koehne, C. punctulata Koehne and some synonyms are lectotypified. The following new nomenclatural combinations are proposed: C. corisperma subsp. hexasperma (Koehne) Duré \& Molero, C. racemosa subsp. longiflora (Koehne) Duré \& Molero and C. racemosa var. ramosior (Koehne) Duré \& Molero. From the chorological point of view, C. micrahtha Humb., Bonpl. \& Kunth, Cuphea rusbyi Lourteig and $C$. sessilifolia Mart. are cited for first time from Paraguay. We also include some taxonomical comments about conflictive taxa like $C$. corisperma Koehne, C. hassleri Koehne and C. racemosa (L. f.) Sprengel, among others.
\end{abstract}

Keywords: Cuphea, Lythraceae, Paraguay, nomenclature, typification, taxonomy, chorology.

\section{RESUMEN}

DURÉ, R. \& J. MOLERo (2000). Notas nomenclaturales, taxonómicas y corológicas sobre el género Cuphea (Lythraceac) en el Paraguay. Collect. Bot. (Barcelona) 25: 225-243.

Se presenta el tratamiento nomenclatural del género Cuphea (Lythraceae) en el Paraguay, que reune el nombre correcto para las 28 especies reconocidas y su correspondiente sinonímia. Para la mayoria de táxones que se mencionan en el texto se ha revisado su tipificación. Se tipifican por primera vez el subgénero Lytrocuphea Koehne y las secciones Brachyandra Koehne y Enatiocuphea Kochne y se lectotipifican C. tuberosa Cham. \& Schltdl., C. inaequalifolia Koehne, $C$. punctulata Koehne, además de varios sinónimos. Se proponen las siguientes combinaciones nomenclaturales: C. corisperma subsp. hexas-

(1) Museo Nacional de Historia Natural del Paraguay. Campus UNA, CDP 2169, San Lorenzo, Paraguay.

(2) Laboratori de Botànica, Facultat de Farmàcia, Universitat de Barcelona, Avda. Joan XXIII s/n. 08028 Barcelona, España. 
perma (Kochne) Duré \& Molero, C. racemosa subsp. longiflora (Kochne) Duré \& Molero y C. racemosa var. ramosior (Kochne) Duré \& Molero. Desde el punto de vista corológico se citan por primera vez para el Paraguay C. micrahtha Humb., Bonpl. \& Kunth, Cuphea rusbyi Lourteig y $C$. sessilifolia Mart. Se incluyen también algunos comentarios taxonómicos sobre táxones críticos, como $C$. corisperma Koehne, $C$. hassleri Kochne y C. racemosa (L. f.) Sprengel, entre otros.

Palabras clave: Cuphea, Lythraceae, Paraguay, nomenclatura, tipificación, taxonomía, corología.

\section{Introducción}

La preparación de las litráceas para la Flora del Paraguay, ha requerido un estudio detallado del género Cuphea, que con 28 especies finalmente reconocidas sobre un total de 38 especies contabilizadas para la familia en el Paraguay, es sin duda el género más diversificado y complejo (DuRÉ, 1999). Paraguay representa la continuidad natural de un núcleo de especiación primario del género que se centra en el Brasil meridional (Minas Geraes y Mato Grosso, principalmente) y es por, tanto, un centro activo de diferenciación, que también se hace extensivo a las zonas fronterizas de Bolivia oriental y Argentina septentrional.

El género ya era relativamente bien conocido en el Paraguay desde finales del siglo XIX y principios del XX, gracias a los trabajos del monógrafo KoEHNE (1903, 1908, 1910a, b, entre otros). Koehne estableció una fructífera colaboración con E. Hassler y pudo acceder a una extensa colección de Cuphea del Paraguay. Paralelamente a los trabajos del monógrafo, HASSLER (1898, 1902, 1909), en colaboración con Chodat, recojen la información que les proporciona Koehne en Plantae Hasslerianae, amplian la distribución de las especies en el Paraguay y describen algunos táxones infraespecíficos. Tras un largo paréntesis, hay que esperar a los trabajos de Lourteig (1943, 1948, 1964, 1969, 1986, 1987 y 1989), para encontrar, directa o indirectamente, nuevas referencias sobre las Cuphea del Paraguay. Recientemente, las intensas herborizaciones de Cuphea efectuadas por los autores de este artículo en todo el Paraguay durante las campañas de los años 1994, 1995 y 1996, han adicionado tres nuevas especies al catálogo y completado sustancialmente su distribución.

En el aspecto nomenclatural, se ha hecho un esfuerzo no despreciable de compilación y revisión de algunos aspectos de tipificación y sinonímia, que completa, para las especies paraguayas, el extenso trabajo previo de Lourteig. El trabajo también ha requerido de algunos cambios nomenclaturales con el fin de adecuar los nombres al criterio taxonómico de los autores. Presentamos a continuación el tratamiento nomenclatural del género Cuphea, que complementará la edición de las Lythraceae para la Flora del Paraguay (Conservatoire et Jardin Botaniques de Genève \& Missouri Botanical Garden), junto a otros comentarios de índole taxonómica y corológica. El orden sistemático aceptado para el género es el de KOEHNE (1903), que sufre pocas modificaciones si se acepta la tipificación del género recientemente propuesta (cf. MOLERO \& ZIJLSTRA, 1999). 


\section{TRATAMIENTO NOMENCLATURAL}

Cuphea P. Browne, Civ. Nat. Hist. Jamaica: 216. 10 Marzo 1756.

Tipo: C. viscosissima Jacq. (designado por J. Swart en la tarjeta ING n ${ }^{\circ}$ 20706, Jun. 1965; cf.: Molero \& ZiJlstRA, 1999).

\section{Subgen. Cuphea}

= Cuphea P. Br. subgen. Eucuphea Koehne, Ind. Sem. Hort. Bot. Berol., appendix altera, 1873. 1874.

$=$ Cuphea P. Br. subgen. Bracteolatae S. A. Graham in Syst. Bot. Monogr. 20: 42. 1988.

Sect. Brachyandra Koehne in Bot. Jahrb. Syst. 2: 137. 1881.

Lectótipo (designado aquí): C. micrantha Humb., Bonpl. \& Kunth.

= Sect. Balsamona subsect. Melaniun Koehne in Mart., Fl. Bras. 13(2): 255. 1877.

Esta sección recoge a la mayor parte de especies autógamas del género de flores pequeñas (4-7 $\mathrm{mm}$ ), poco zigomorfas, con estambres insertos hacia la mitad de tubo y anteras inclusas que se sitúan muy próximas al estigma. No obstante GraHAM (1998), sobre la base de un análisis cladístico que engloba prácticamente a la totalidad de las especies de la sección, considera que se trata de un grupo no natural (polifilético), que incluye a muchos representantes que están más relacionados con otras secciones del género. Las similitudes en la estructura floral entre las especies de esta sección se deberían más a un proceso de convergencia adaptativa desde la alogamia hacia la autogamia, que a un auténtico parentesco. Desde el punto de vista estrictamente morfológico, algunas especies como $C$. rusbyi, muestra algún carácter de transición hacia la sección Euandra, como la línea de inserción de los estambres que alcanza casi el tercio superior del tubo; esta especie también muestra un gran parecido con C. corisperma Koehne (de la sección Euandra) en hábito y otros caracteres vegetativos, aunque difiera en los caracteres florales.

1. Cuphea calophylla Cham. \& Schltdl. subsp. mesostemon (Koehne) Lourteig in Sellowia 16: 131-132. 1964.

$\equiv$ C. mesostemon Koehne in Mart., Fl. Bras. 13(2): 252, lám. 43, f. 5. 1877. Indicatio locotypica: «Habitat in provincie Minas Geraes et S. Paulo, e. gr. in campis Prope Ouro Preto: Martius; ad Taguassú: Lund n. 997; in humidis umbrosis prope Lorena: Riedel n. 1595; locis carundem provinciarum ocurridas non indicantes: Sello n. 5090, Guillermina n. 352, Barcal n. 3887». Tipo: "Brasil meridional, leg. Sello" (LourTEIG, 1969: 27). Nos parece una tipificación claramente insuficiente. No hemos podido acceder a ninguna de las exsiccatas del síntipo que menciona Koehne, por lo que queda pendiente la correcta tipificación de este taxon.

$=C$. mesostemon Koehne var. missionera Lourteig in Lilloa 9: 352. 1943. Ind . loc.: "Misiones, San Ignacio». Holótipo en LP!: "Flora del Territorio de Misiones / República Argentina / San Ignacio / 11-XII-1941 / Birabén 5297” (LourTEIG, l. c.). 
2. Cuphea micrantha Humb., Bonpl. \& Kunth, Nov. Gen. Sp. 6 (26): 196. 1823 (Fig. 1).

Ind. loc.: «Crescit ad ripam Orinoci, prope Santa Barbara et juxta San Carlos de Rio Negro». Tipo en HB-P: Humbolt \& Bonpland 1119 (Microficha $n^{\circ} 149$, vidit in MA).

Novedad para la Flora del Paraguay. Se distribuye desde México y el Caribe hasta el centro y este del Brasil (Piauí, Bahía, Goiás, Minas Geraes). En el Paraguay alcanza su límite meridional de distribución, rara y escasa en la zona oriental, en los Departamentos de Concepción y Amambay. Vive en pastizales y campos cerrados o abiertos de suelo arenoso.

Material estudiado. - Concepción: "Karanza Bola, camino a Paso Mbutú"

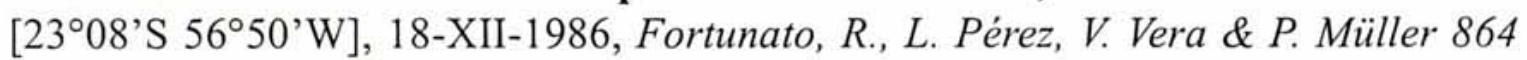
(PY 8133); “Loreto, a 5 km camino a San Luís" [2314'12"S 57²1'03”W], 23-XI1996, Molero, J. \& R. Duré 1040 (BCF, P, PY). Amambay: "Parque Nacional Cerro Corá" [22³9'S 5603'W], 8. II. 1982, Solomon, J. C. \& al. 6834 (MO 3515424, PY); "Parque Nacional Cerro Corá, límite oeste del Parque" [22³9'S 5603'W], 7-I-1988, Soria, N. \& E. Zardini 1958 (CTES 134600).

3. Cuphea carthagenense (Jacq.) Macbr. in Field Mus. Nat. Hist. Chicago 8 (2): 124. 1930.

$\equiv$ Lythrum carthagenense Jacq., Enum. Syst. P1.: 22. 1760 (sin indicación de localidad); Sel. Stirp. Amer. Hist.: 148. 1763. Ind. loc.: «Habitat Carthagenae in sylvis umbrosis \& humidiusculis» Material tipo: según nos comunica el Dr. Vitek, no existe material tipo de este taxon en W-JAQC. Respecto a la colección de plantas de Jacquin que se conserva en BM-LINN, en donde pudiera hallarse material tipo de este taxon (cf.: D'ARCY, 1970), el Dr. Jarvis nos comunica que el material de Cuphea del Nuevo Mundo procedente de este herbario ha sido cedido en préstamo a US, por lo que no ha sido posible consultarlo.

= Balsamona pinto Vandel., Fasc. Pl.: 15, lám. 3. 1771. Ind. loc.: «Habitat in Brasilia». Lectótipo (designado aquí): [Icon] Balsamona pinto Vandel., Fasc. Pl.: 15, lám. 3. 1771. [三 Parsonsia pinto (Vandel.) Heller, Minnes. Bot. Stud. 9: 862. 1897]. No hemos encontrado material tipo de Vandelli ni en LISU ni en LINN, por eso tipificamos sobre la lámina.

$=$ C. balsamona Cham. \& Schltdl. in Linnaea 2: 363. 1827. Ind. loc.: «In humidis provinciae Rio de Janeiro Brasiliae». Tipo: «Rio de Janeiro, Beyrich s. n., sept. 1822» (LOURTEIG, 1989: 23, sin especificar herbario), n. v.

Rescatamos del olvido el protólogo prioritario de Lythrum carthagenense (Jacquin, 1760) sobre el que suele utilizarse habitualmente (Jacquin, 1763) [cf.: Macbride, l. c.; Lourteig, 1989; Graham, 1990].

4. Cuphea rusbyi Lourteigin Svensk. Bot. Tidskr. 48 (1): 84-85. 1954 (Fig. 2).

Ind. loc.: "Bolivia, dep. La Paz, Rurrenabaque, alt 1000 ft., leg. H. H. Rusby (117 a), 1921 ». Holótipo en NY(!): "Bolivia / Rurrenabaque / 1000 ft. / H. H. Rusby 117a / 1921"; pliego conteniendo un único ejemplar. Isótipo en US.

Descrita originariamente de Bolivia, de Rurrenabaque y Cochabamba, entre 350$1000 \mathrm{~m}$ de altitud, en un ambiente presumiblemente húmedo (aunque la transcripción 


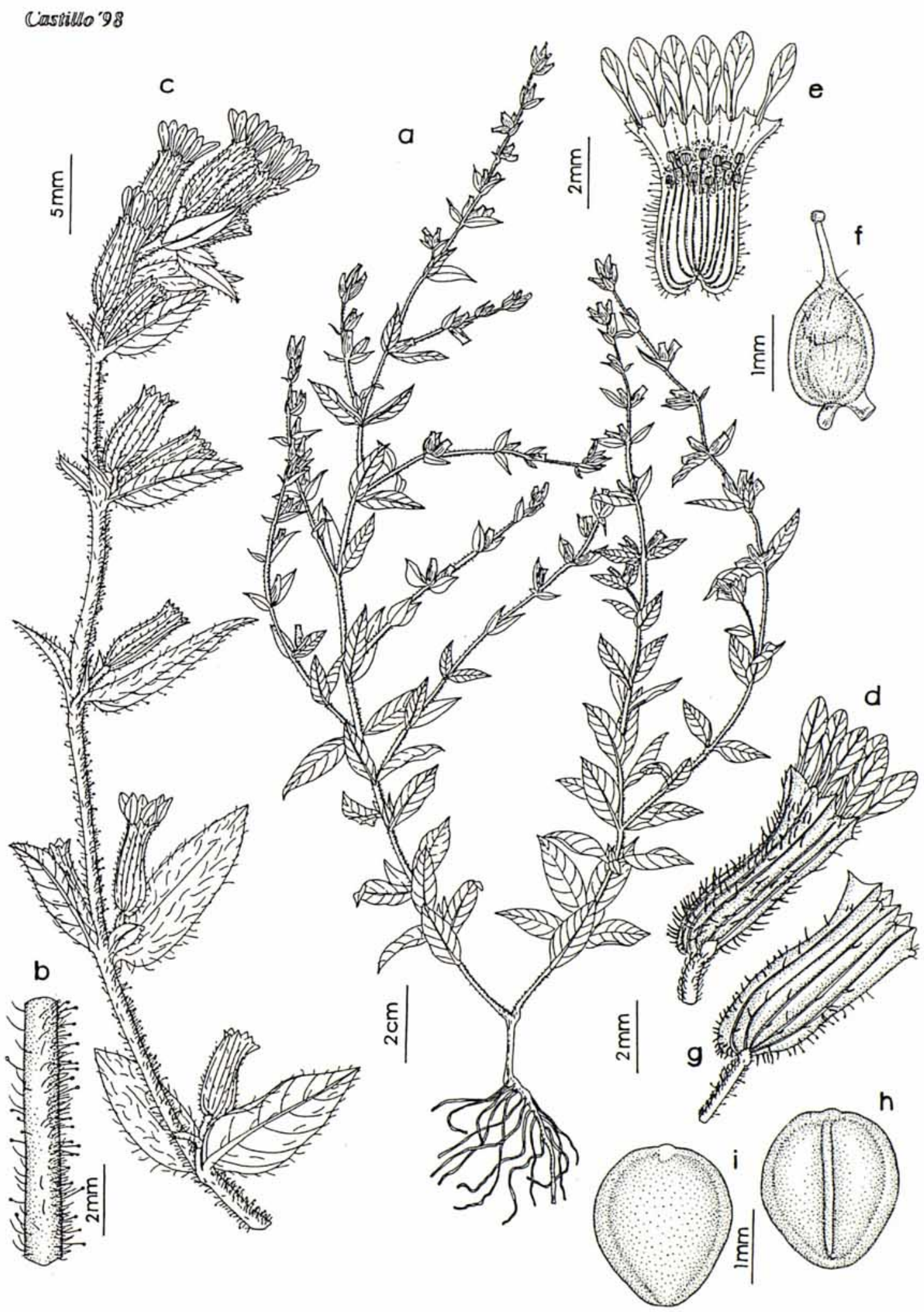

Fig. 1 Cuphea micrantha (Molero \& Duré 1040): a) hábito; b) detalle de un segmento de la zona media del tallo; c) rama florifera; d) flor; e) flor abierta; f) gineceo y disco; g) hipanto fructificado; h) semilla en visión ventral; i) semilla en visión dorsal. 
de las etiquetas no aclara ese aspecto), su área alcanza, hacia el este, el Paraguay. Localizada en los Departamentos de Concepción y Alto Paraguay, aunque pudiera hallarse también en el territorio brasileño próximo. Vive en campos, terraplenes y márgenes de pistas, sobre suelos arenosos preferentemente secos.

Material estudiado. - Concepción: "Paso Horqueta, a $6 \mathrm{Km}$ camino a San Luís" [2259'08'S 57²1'50”'W], 23-XI-1996, Molero, J. \& R. Duré 1047 (PY18795); "Entre San Alfredo y Paso Horqueta, a $15 \mathrm{Km}$ antes de Paso Horqueta" [2310'S 57²0’W], 20-III-1998, Pérez de Molas, L., M. Quintana \& L. Spinzi 3398 (PY). Alto Paraguay: "Chaco Paraguayo: F. Olimpo" [2100'S 5750’W], 18-XI-1946, Rojas, T. 13806 (CTES 52244, MAB).

Endemismo centro sudamericano de área muy restringida, que se cita aquí por primera vez del Paraguay. Los materiales paraguayos presentan algunas pequeñas diferencias respecto de los bolivianos, que no nos atrevemos a valorar como diagnósticos: ejemplares claramente anuales, con las hojas más cortas y más anchamente elípticas que los de Bolivia, casi lineares; las bractéolas son glabras (ciliadas en los materiales bolivianos); los tubos florales verdosos (purpurescentes en las plantas bolivianas), y el disco reflejo lingüiforme (subhorizontal y lobado superiormente en los ejemplares bolivianos). Los restantes caracteres coinciden en lo esencial.

Sect. Euandra Koehne in Bot. Jahrb. Syst. 2: 146. 1881.

= Sect. Balsamona subsect. Eubalsamona, ser. 2, 3. Koehne in Mart., Fl. Bras. 13(2): 266. 1877.

$=$ Sect. Balsamona subsect. Hilairea Koehne in Mart., Fl. Bras. 13(2): 266. 1877.

Sin duda la sección más extensa, que reúne a táxones muy diversos, por lo que es difícil seleccionar caracteres comunes. En general reúne a especies alógamas claramente zigomorfas con tubos florales medianos [(5)7-12(15) $\mathrm{mm}$ ] y estambres insertos entre el $1 / 3$ y el $1 / 4$ superior del tubo floral, con anteras que igualan 0 superan los lóbulos calicinales. C. strigulosa muestra nexos de unión con la sección Brachyandra por su parecido con C. calophylla, por la línea de inserción de los estambres algunas veces por debajo del $1 / 3$ superior del tubo y por las anteras prácticamente inclusas, excepto las de los estambres ventrales. Esta especie y $C$. rusbyi (que presenta la línea de inserción de los estambres más elevada de su sección), rompen la discontinuidad morfológica entre las secciones Euandra y Brachyandra, justificando tal vez mantener la más amplia sección Balsamona Koehne, que fue descrita en la Flora del Brasil y posteriormente desmembrada por el mismo Koehne.

5. Cuphea strigulosa Humb., Bonpl. \& Kunth, Nov. Gen. Sp. 6: 204. 1824.

Ind. loc.: «Crescit prope Ibague, ad radices Andium Quiducnsicem, alt. 700 hex.» Holótipo en HB-P: “ Humboldt \& Bonpland 1803, Octobri” (Lourteig, 1989: 23), n. v. Isótipos en B-W, H (según LourTeiG, l. c.), n. v.

$\equiv$ C. strigulosa Humb., Bonpl. \& Kunth subsp. opaca Koehne in Mart., Fl. Bras. 13(2): 257. 1877, nom. illeg. (Basado en el mismo tipo que la especie). 


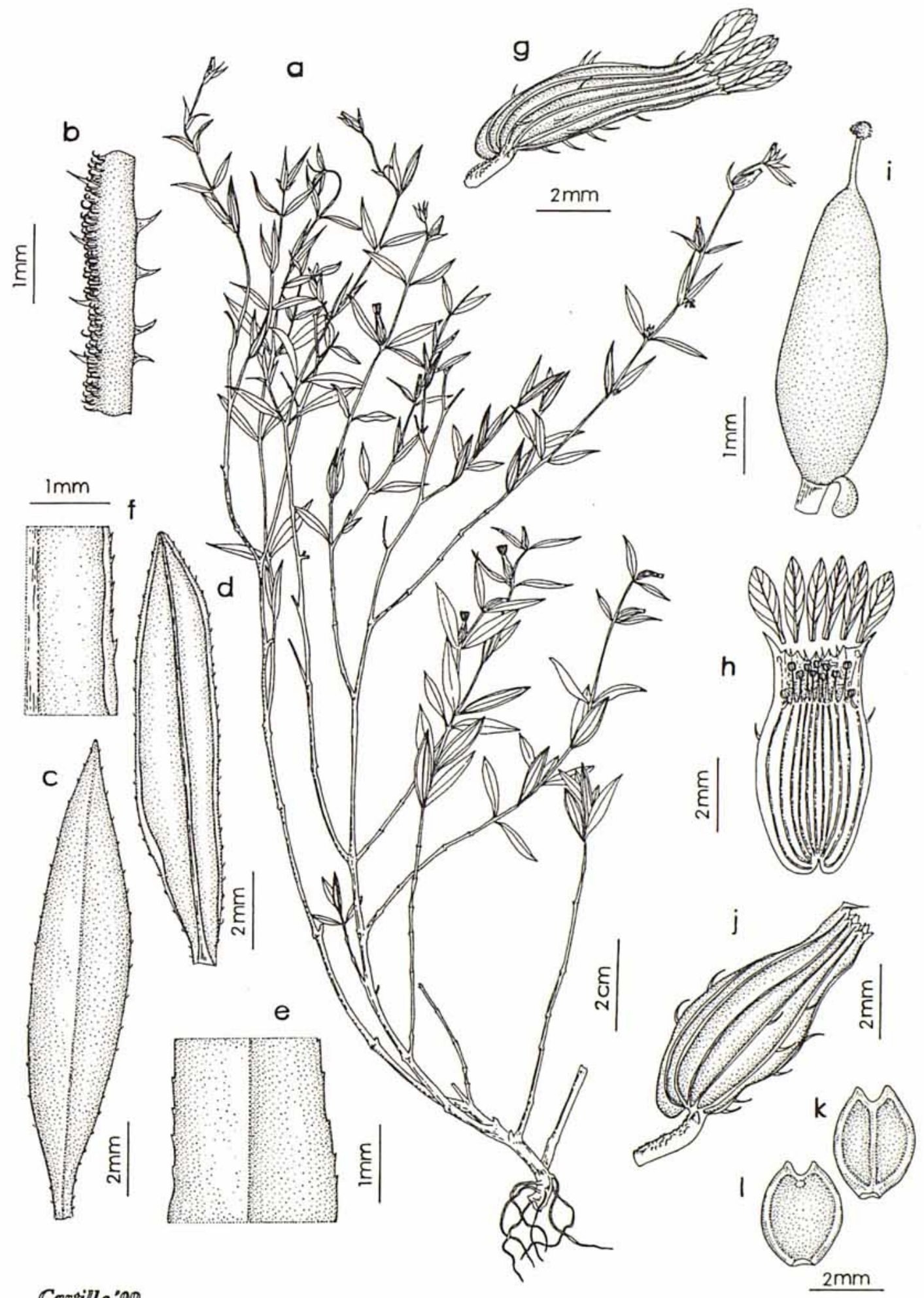

Cossillo'99

Fig. 2. Cuphea rusbyi (Molero \& Duré 1047): a) hábito; b) detalle de un segmento de la zona media del tallo; c y e) hoja y un detalle de la cara adaxial; d y f) hoja y un detalle de la cara abaxial; g) flor; h) flor abierta; i) gineceo y disco; j) hipanto fructificado; k) semilla en visión ventral; l) semilla en visión dorsal. 
$=$ C. strigulosa Humb., Bonpl. \& Kunth subsp. nitens Koehne in Mart., Fl. Bras. 13(2): 257. 1877. Ind. loc.: «Habitat in Brasiliae prov. Bahía et Rio de Janeiro: Sello n. 137, Martius Hb. Fl. Bras. n. 422 et 423 (ad Ilheos), Riedel n. 339 (ibidem) Harrison et Paulo Barbosa (Rio de Janeiro), Sieber Fl. nixta n. 383». Tipo en P: “Martius 422» (LOURTEIG, 1987: 24), n. v.

$=$ C. strigulosa Humb., Bonpl. \& Kunth subsp. opaca Koehne var. sinclairii Koehne in Engler, Pflanzenr. 17 (IV. 216): 124. 1903. Ind. loc.: "Andines Südamerika: Ecuador (Sinclair)». Tipo: "Ecuador, Sinclair" ( según LouRTEIG, 1987: 10; 1989: 24: “v. Koehne, Atlas ined., analysis”), n. v.

= Cuphea fiebrigii Koehne in Feddes Repert. Spec. Nov. Regni Veg. 8: 166-167. 1910. Ind. loc.: « $22^{\circ}-23^{\circ}$ lat., zwischen Río Apa und Aquidabán, bei Caballero-cué, auf feuchtem Camp bei Buschwerk». Holótipo en G!: "Nördl. Paraguay (22-23ำ lat.) / zwischen Río Apa und Aquidabán / bei Caballero-cué / feuchtem Camp / bei Buschwerk / Febr. / Fiebrig 4727' (LourTEIG, 1987: 10). Isótipos en AS!, G!, BM!, $\mathrm{P}$ ! y $\mathrm{K}$ !.

6. Cuphea campylocentra Griseb., Symb. Fl. Argent.: 130. 1879.

Ind. loc.: «E., pr. Concepción del Uruguay». Tipo en GOET: "Lorentz 545, p. p., 1876» (LOURTEIG, 1969: 44, tipificación claramente insuficiente), n. v. Según esta autora, también material tipo en BAF, n. v.

$=$ Cuphea grata Koehne in Feddes Repert. Spec. Nov. Regni Veg. 8: 165. 1910. Ind. loc.: «Paraguay, $22^{\circ}-23^{\circ} \mathrm{s}$. Br., zwischen Rio Apa und Aquidabán bei Caballerocué». Holótipo en G!: "Nördl. Paraguay (22-23 lat.) / zwischen Río Apa und Aquidabán / bei Caballero-cué / Camp bei ufemald, (feuchtem) / Febr. / Fiebrig 4807» (Lourteig (1987: 11). Isótipos en AS!, BM!, G! y P!.

7. Cuphea corisperma Koehne in Bot. Jahrb. Syst. 41: 85-86. 1908.

Ind. loc.: «Paraguay, in viciniis Caaguazú». Holótipo en G!: Prope Caaguazú in argilosis / II-1905 / Hassler 9031 ( LourTEIG, 1987: 11). Isótipos en BM!, G!, MO!, P!, W!.

7a. C. corisperma Koehne subsp. hexasperma (Koehne) Duré \& Molero, comb. \& st. nov.

$\equiv$ Cuphea hexasperma Koehne in Feddes Repert. Spec. Nov. Regni Veg. 8: 165166. 1910. Ind. loc.: «Nördlicher Chaco, Puerto Olimpo, Dec., Fiebrig pl. paraguarienses N $\mathrm{N}^{\circ}$ 477». Holótipo en G!: "Chaco septentrionalis: Fuerte Olimpo / XII-1907 / Fiebrig 1477». LOURTEIG, 1987: 11, carpeta con cinco pliegos, con etiqueta de identificación de Koehne. Isótipos en G! y S!.

C. corisperma y C. hexasperma son consideradas meros sinónimos de C. campylocentra por LourTEIG (1987). Pero se trata de tres táxones independientes que pueden diferenciarse sin excesivos problemas. C. campylocentra difiere de las dos primeras principalmente por el hábito, no ramificado o muy escasamente en la base, $\mathrm{y}$ por el tipo de indumento y mayor tamaño del tubo floral (9-12 x 2-3 mm), mientras que $C$. corisperma presenta tallos muy ramificados en la base y tubos florales peque- 
ños $(8,0-11 \times 1,4-2,0 \mathrm{~mm})$. Dentro de esta última especie, la subespecie hexasperma difiere por sus pétalos ungiculados y estilo glabrescente de la subespecie corisperma, con pétalos desprovistos de uña y estilo glabro. Como ocurre con otras especies de Cuphea, es más difícil concretar estas diferencias entre C. campylocentra y $C$. corisperma sobre el papel, que la evidencia que surge de la comparación en fresco y de la consulta de los materiales de herbario.

8. Cuphea glutinosa Cham. \& Schltdl. in Linnaea 2: 369. 1827.

Ind. loc.: «Hanc uti videtur vulgarem in Brasilia meridionali plantam plurimis locis (Salto, Rincon das galhinas, etc.) magna in copia leg. Sello». Material tipo en W! y en P!: "Herb. Reg. Berolinense / C. glutinosa Cham. \& Schltdl. / Brasilia, Sello".

- C. thymoides Griseb., Symb. Fl. Argent.: 130. 1879 [non Cham. \& Schltdl. in Linnaea 2: 368. 1827].

9. Cuphea ingrata Cham. \& Schltdl. in Linnaea 2: 371-372. 1827.

Ind. loc.: «In Brasilia aequinoctiali legerunt Sello et Beyrich». Tipo: "Brasil, Rio Paquaquer, Beyrich" (LourTEIG, 1969: 35, sin especificar herbario), n. v.

$=$ C. ingrata var. grandifolia A. St.-Hil., Fl. Bras. Merid. 3: 107-108. 1833. Ind. $l o c .:$ «Octobre ad ripas rivi Ubà, provincià Rio de Janeiro». Holótipo en P!: "Brasil, Minas Geraes / griny humidis près Retiro norte de Rio de Janeiro à Villa Rica / voyage de 1816 à 1821 / A. Saint Hilaire 248"; pliego que contiene un único ejemplar.

$=C$. ingrata var. parvifolia A. St.-Hil., Fl. Bras. Merid. 3: 107-108. 1833. Ind. loc.: «In provinciis Rio de Janeiro \& Minas Geraes, regione sylvarum, haud infrequens». Holótipo en P!: "Brasil, Minas Geraes / près la cascada de Villa Rica en janvier / voyage de 1816 à 1821/ A. Saint Hilaire 170”; el pliego incluye 2 ejemplares.

10. Cuphea thymoides Cham. \& Schltdl. var. saturejoides [«satureioides»] A. St.Hil., Fl. Bras. Merid. 3: 83. 1833.

Ind. loc.: «Brasil, Minas Geraes, Serra da Ibitipoca». Holótipo: en P!: "Sierra da Ibitipoca / prov. de Minas, A. St.-Hilaire 540" (LourTEIG, 1969: 41).

$=C$. mesostemon forma angustifolia Chodat in Bull. Herb. Boissier 6: 72. 1898. $\equiv$ C. phoenix Koehne in Engl. in Bot. Jahrb. Syst. 41: 84-85. 1908. Ind. loc.: «In arenosis pr. rivulum Carapeguá». Holótipo en G!: "Plantae Paraguarienses / In arenosis pr. riv. Carapeguá / Oct. / Hassler 1219” (LourTEIG, 1964: 133). Isótipos en G (7040 / 186: pliego con dos ejemplares, el isótipo es el ejemplar de la izquierda, el de la derecha correponde a $C$. calophylla subsp. mesostemon) y en P!.

11. Cuphea linarioides Cham. \& Schltdl. in Linnaea 2: 367-368. 1827.

Ind. loc.: «In Brasilia aequinoctiali legit Sello". Material tipo en W!: Herb. Reg. Berolinense / Brasilia, Sello 4999.

$=C$. linarioides var. communis A. St.-Hil., Fl. Bras. Merid. 3: 116. 1833. Ind. loc.: «In campis partis occidentalis provinciae Minas Geraes dictae Sertao». Holótipo en P!: "pastagem près la fazenda d'Olho d'Agua, Sertao do Minas, A. Saint-Hilaire"; pliego que contiene 7 ejemplares pequeños subherbáceos. 
$=C$. linarioides var. minutifolia A. St.-Hil., Fl. Bras. Merid. 3: 117. 1833. Ind. loc.: «In campis partis occidentalis provinciae Minas Geraes dictae Sertao». Holótipo en P!: "Pastagen près Contendas dans le / Sertao das Minas, donné per Ms. Antonio Noguera Duarte / voyage de 1816 à 1821 / A. Saint-Hilaire”; el pliego incluye 6 ejemplares pequeños subherbáceos.

$=$ C. linarioides var. nana A. St.-Hil., Fl. Bras. Merid. 3: 117. 1833. Ind. loc.: «In campis nuperrimè crematis lucitanicè Queimadas». Holótipo en P: "Un pastagem recemment boreté / près Toroba dans le Sertao / de Minas. . . / voyage de 1816 à 1821 / A. Saint-Hilaire 1733 bis" .

$=C$. linarioides var. crassiuscula A. St.-Hil., Fl. Bras. Merid. 3: 117. 1833. Ind . loc.: «In campis arenosis prope Registro Velho, parte australi altâque provinciae S. Pauli, vulgò Campos Geraes». Holótipo en P!: "Pasturagem tablonem près Registro / Velho, midi de Campos Geraes. . . / voyage de 1816 à 1821 / A. Saint-Hilaire 1320 bis. "; el pliego contiene 2 ejemplares.

$=$ C. guelichii Speg. in Anal. Soc. Cient. Arg. 16: 99. 1883. Ind. loc.: «Hab. in pratis 'Campo chico' vocatum ad ripam sinistram fluminis 'Yacan-guazú' in provincia Argentina Misiones, Maj. 1883, leg. Cl. Carolus v. Gülich.» Lectótipo en LP 14595! (designado aquí): "Campo chico / lado izqz. del Yacanguazú / Fl. Colorata”, sin recolector, ni fecha. Pliego conteniendo 5 ejemplares.

- ? Lythrum calcaratum Vellozo, Fl. Flum. 5: 200, lám. 7. 1825.

13. Cuphea confertiflora A. St.-Hil., Fl. Bras. Merid. 3: 112-113. 1833.

Ind. loc.: «Lecta ad margines viarum in campis prope amnen Hyapó, australi altâque prov. S. Pauli, vulgò Campos Geraes, Florebat Februario». Holótipo en P!: "Brasil, Sao Paulo, Campos Geraes, Hyapó, A. Saint-Hilaire, 1816-21" (LourTeIG, 1969: 53).

= C. tuberosiformis Koehne ex Bacigalupi, Contr. Gary Herb. Harvad Univ. 95: 16-17. 1931. Ind. loc.: "Brasil: Paraná, Tamanduá, in campo", Feb. 24, 1910. Holótipo en S!: P. Dusen s/n. Isótipo en G!.

14. Cuphea lysimachioides Cham. \& Schltdl. in Linnaea 2: 374-376. 1827.

Ind. loc.: «Per totam Brasiliam aequinoctialem ac meridionalem (ex. gr. Estrella das Tombas) leg. Sello». Tipo: no designado. Como LourTEIG (1987: 22), tampoco hemos hallado material tipo de Sello.

$=C$. lysimachioides forma brevipes Koehne in Bot. Jahrb. Syst. 2: 151-152. 1882. Ind. loc.: «Paraguay: Itaigu, près de Villa-Rica, sur le bord des mares». Holótipo en P!: "Itaugu, près de Villa-Rica, sur le bord des mares / 1 février 1876 / B. Balansa 2211 c"; pliego que incluye 5 ejemplares, escogemos como tipo el ejemplar de la izquierda (LourTEIG, 1987: 22, ut C. pterosperma var. brevipes Koehne).

$=$ C. lysimachioides var. crassifolia Chodat in Bull. Herb. Boissier VI. App. 1: 73. 1898. Ind. loc.: Paraguay: «In campo prope Paraguari, fl. Jan. (uniq.)». Material tipo: Hassler 142, no encontrado.

$=C$. lysimachioides var. villosa Koehne in Feddes Repert. Spec. Nov. Regni Veg. 8: 196. 1910. Ind. loc.: «Nord-Paraguay: 22-23 s. Br., zwischen Rio Apa und Aquidabán, Caballero-cué, nasser Camp, Feb.». Holótipo en G!: "Nördl. Paraguay: 
22-23 lat. / zwischen Rio Apa und Aquidabán, Caballero-cué / nasser Camp / 12 Febr. / Fiebrig 4923" (LourTEIG, 1987: 22). Isótipos en AS!, G! y K!.

= Cuphea hassleri Koehne in Bull. Herb. Boissier 2. sér. 4: 401. 1902, syn. nov. Ind. loc.: «Paraguay: prope Igatimi. Hassler 4802». Holótipo en G!: "In campis pr. Ygatimí / X-1898-99 / Hassler 4802 (unicum)".

$=C$. lysimachioides var. dubia Koehne in Feddes Repert. Spec. Nov. Regni Veg. 8: 196-197. 1910. Ind. loc.: «Nord-Paraguay: An demselben Standort wie vor. Fiebrig 4715 und 4715a (Herb. Hassler)». Holótipo en G!: "Nördl. Paraguay: 22-23 lat. / zwischen Rio Apa und Aquidaban / Caballero-cué / Febr. / Fiebrig 4715"; pliego que contiene un único ejemplar (LOURTEIG, 1987: 22).

C. hassleri fue descrita por Koehne sobre la base de un único pliego (Hassler 4802 in $\mathrm{G})$, que contiene un único ejemplar. Según nuestro criterio este ejemplar entra perfectamente en la amplia variabilidad no fijada genéticamente que muestra $C$. lysimachioides. Las escasas diferencias respecto a los ejemplares más típicos se basan en el indumento escasamente villoso de la superficie interna del hipanto y de los filamentos estaminales, que en aquellos son más densamente villosos; no obstante, otros materiales estudiados de C. lysimachioides (p. ej.: Hassler 4262 in G) presentan también esta característica, aunque no otras consideradas diagnósticas; también KoEHNE atribuye 8 primordios seminales a este único ejemplar (difícilmente demostrable dado el estado de floración temprana del mismo), pero C. lysimachioides presenta en el Paraguay de 6-10 primordios seminales, por lo que no debe considerarse este un rasgo distintivo. Algo más tarde, KoEHNE (1903) identifica como C. hassleri un pliego depositado en $\mathrm{G}$ (Hassler 7869). En realidad se trata de un material claramente distinto al anterior por numerosos caracteres: hábito, inserción del disco, presencia de vesículas, indumento medifijo externo, y superficie interna del hipanto (glabra en la zona de los estambres ventrales, frente a villosa en $C$. lysimachioides). Este pliego debe atribuirse sin duda a C. pterosperma; aunque represente un extremo de variación de esta especie por su indumento densamente glandular; no obstante, presenta los inconfundibles tricomas medifijos. En la clave de su monografia, KoEHNE (1903) mezcló características de estas dos recolecciones, atribuyendo finalmente a $C$. hassleri unas características que no responden a un taxon real. En definitiva, el taxon $C$. hassleri basado en la descripción original, debe ser considerado mero sinónimo de C. lysimachioides.

15. Cuphea pterosperma Koehne in Mart., Fl. Bras. 13(2): 264-265, t. 47, f. 5. 1877. Ind. loc.: «Habitat in Brasilia: Weddell n. 1792, et in Paraguay: Rengger». Lectótipo en P!: «Brasil, Minas Geraes, Abaité, Weddell 1792, XI-1843» (LouRTEIG, 1964: 141).

= C. pterosperma var. cuneata Koehne in Bot. Jahrb. Syst. 41: 86. 1908. Ind. loc.: «Paraguay: Caaguazú (Hassler n 9411)». Holótipo en G!: «Plantae Paraguarienses / Prope Caaguazú in argillosis humidis / Sept. / Hassler 9411 (unicum)».

$=C$. gracilis var. brasiliensis A. St.-Hil., Fl. Bras. Merid. 3: 101. 1833. Ind. loc.: «Lecta in prov. S. Pauli». Holótipo en P!: "Brésil / (ex herb. Lusitan.), Ferreire, sin fecha"; pliego que contiene 12 ejemplares (LOURTEIG, 1987: 22).

16. Cuphea stenopetala Koehne in Engl. in Bot. Jahrb. Syst. 2: 150. 1881. 
Ind. loc.: «In planitiebus humidis. Bras. extr. Paraguay: Caaguazú mart.». Holótipo en G!: "Caaguazú, dans les prairies humides / 20-III-1874 / B. Balansa 2212”. Isótipos en G! y P!.

17. Cuphea tuberosa Cham. \& Schltdl. in Linnaea 2: 372. 1827.

Ind. loc.: «In provinciis meridionalibus Brasiliae legit Sello». Lectótipo (designado aquí) en G!: "Cuphea tuberosa Nob. / In Brasilia meridionalis / Sello”. Material anotado por Chamisso.

Sect. Trispermum Koehne in Mart., Fl. Bras. 13(2): 283. 1877.

Lectótipo: C. antisyphylitica Humb., Bonpl. \& Kunth (designado por LourTEIG, 1987: 26).

18. Cuphea flava Spreng. In Nov. Prov.: 14. 1819.

Ind. loc.: "Brasil, cul. Jardín Hale ex herb. Sprengel". Material tipo: según Lourteig (1987: 39) Sprengel envió una parte de su especimen a Balbis, de Turín. Probablemente en TOR se conserve material tipo, que no hemos visto.

19. Cuphea inaequalifolia Koehne in Mart., Fl. Bras. 13: 286, t. 51, f. 6. 1877.

Ind. loc.: «Habitat in Brasilia occidentali, Tamberlik». Lectótipo (designado aquí) en W!: «Pl. Bras. occid. / Tamberlik s. n.»; pliego que contiene un único ejemplar.

20. Cuphea sessilifolia Mart., Flora 24, Béibl. 2: 7. 1841 (Fig. 3).

Ind. loc.: «Prov. Bahiensis. Octobri. Dryas». Holótipo en BR: "Brasil, Bahía, Martius 421 p. p.” (LourTEIG, 1987: 31, tipificación claramente insuficiente), n. v. Isótipo en $\mathrm{P}$ !.

$=C$. sessilifolia Mart. var. pilosa Koehne forma major Koehne in Mart., Fl. Bras. 13(2): 290-291. 1877. Ind. loc.: «Habitat in provincia Bahía. Martius Hb. Fl. Bras. $n$. 421 partim, Blanchet $n$. 1280, 3217, 3217A». Material tipo: no encontrado, probablemente destruido.

$=$ C. sessilifolia Mart. var. pilosa Koehne forma minor Koehne in Mart., Fl. Bras. 13(2): 290-291. 1877. Ind. loc.: «Habitat in provincia Bahía. Blanchet 1727». Holótipo en G! “ Bresil, Bahía, Blanchet 1727. 1834”.

$=$ C. implexa Koehne in Engl., Pflanzenr. 17(IV. 216): 143. 1903. Ind. loc.: «Brasilien: Piauhy (Gardner n. 2844)». Holótipo en K: Gardner 2844 (LOURTEIG, 1987: 31), n. v.

Considerada hasta ahora endemismo del norte y centro de Brasil (Ceará, Piauí, Bahía, Goiás, Mato Grosso, Distrito Federal, Minas Geraes), se indica aquí por primera vez para el Paraguay. Muy rara y localmente abundante en el Departamento de Canindeyú. Suele hallarse entre pastizales bajos, sobre suelos muy húmedos, agrupándose en colonias dispersas.

Material estudiado. - Canindeyú: "Ygatimí, a $8 \mathrm{Km}$ con destino a Asunción" [2410’'S 5540’ W], 15-XII-1995, Duré, R., A. Bogado \& A. Fleitas 785 (BCF, PY); "Ygatimí, a $8 \mathrm{Km}$ con destino a Asunción" [24¹0'S 5540’W], 15-XII-1995, Duré, R., A. Bogado \& A. Fleitas 786 (BCF, P, PY); "Ygatimí, a $8 \mathrm{Km}$ con destino a Asunción" [2410'S 5540'W], 15-XII-1995, Duré, R., A. Bogado \& A. Fleitas 787 (BCF, PY). 


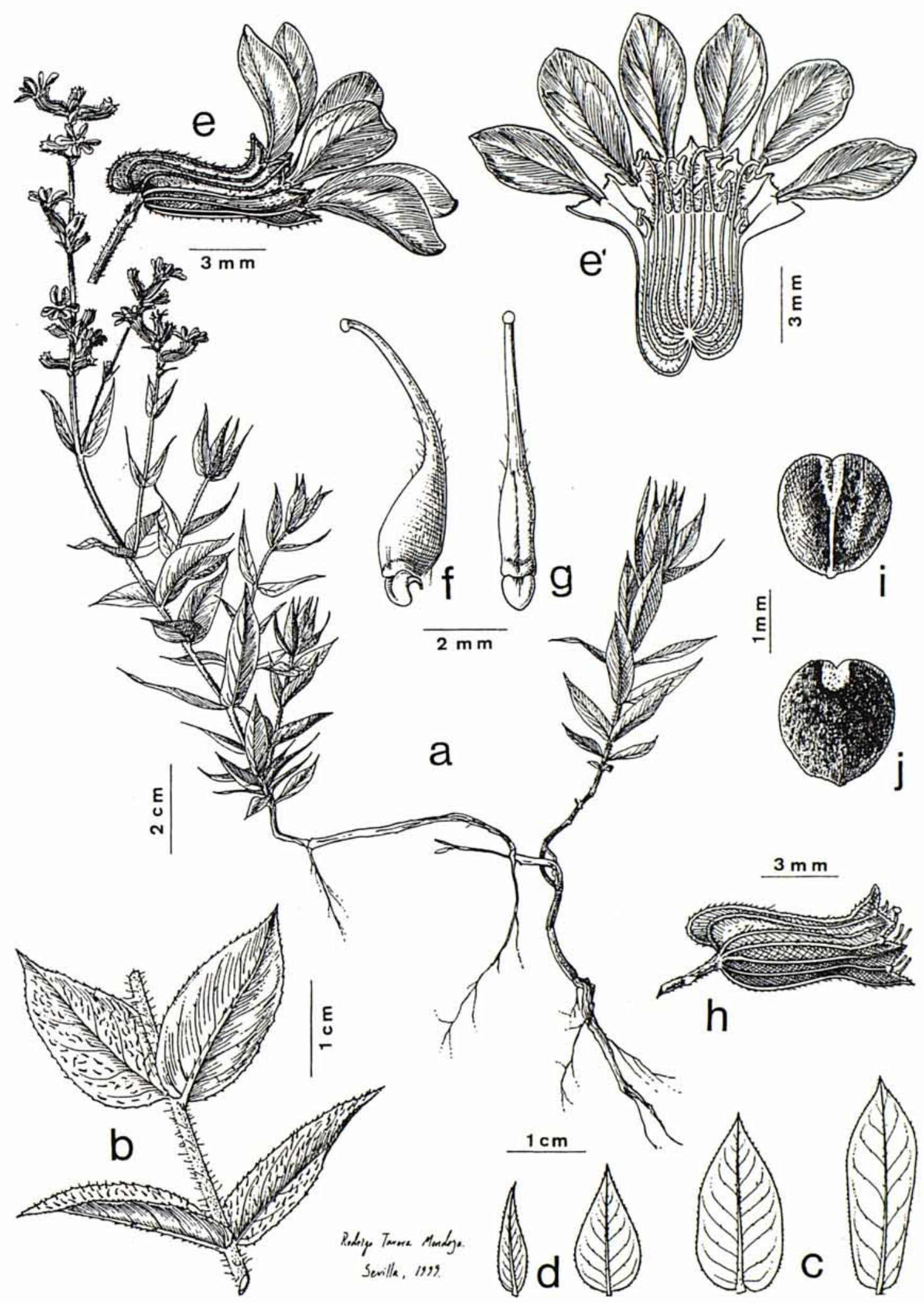

Fig. 3. Cuphea sessilifolia (Duré \& al. 786): a) hábito; b) fragmento de una rama mostrando la disposición de las hojas; c) brácteas; d) hojas basales; e) flor; e') flor abierta; f) gineceo y disco en visión lateral; g) gineceo y disco en visión frontal; h) hipanto fructificado; i) semilla en visión ventral; j) semilla en visión dorsal. 
Sect. Pseudocircaea Koehne in Mart., Fl. Bras. 13(2): 294. 1877.

Lectótipo: C. impatientifolia A. St.-Hil. (designado por LOURTEIG, 1986: 44).

21. Cuphea persistens Koehne in Verhand. Bot. Ver. Brandenburg 30: 277-278. 1888.

Ind. loc.: «Argentina, Tucumán». Holótipo en GOET: "Sauciliaro, Lorentz et Hieronymus, s. n., I-1874" (LOURTEIG, 1986: 50), n. v.

$=C$. mesostemon var. ovalifolia Chodat in Bull. Herb. Boissier 7: 72. 1898. $\equiv C$. ovalifolia (Chodat) Koehne in Bull. Herb. Boissier ser. 2, 4: 401. 1902. Ind. loc.: «In campo vicin. lag. Ypacaray, fl. Aug. $n^{\circ} 874$ (unic.); id. In campo prope Cordillera de Altos, fl. Jan., 1810; in campo pr. Tucanguá, fl. sept. $n^{\circ} 905$; in campo pr. Cordillera de Altos, fl. jul., n 571». Lectótipo en G: "Laguna de Ypacaray, Hassler 874" (designado por LOURTEIG, 1986: 50).

$=C$. persistens Koehne var. formosana Lourteig in Darwiniana 8: 271-272. 1948. Ind. loc.: «Argentina, Formosa, Pilcomayo, $3 \mathrm{Km}$ de Porteñito, al N». Holótipo en LIL: “Morel 3869, 6-X-1947” (LourTEIG, 1. c.), n. v.

22. Cuphea sessiliflora A. St.-Hil., Fl. Bras. Merid. 3: 110. 1833.

Ind. loc.: «Lecta prope vicum Contendas in parte desertâ occidentalique provinciae Minas Geraes dictâ Sertao et a venerando parocho Antonio Noguera Duarte communicata». Holótipo en P!: "Près le village de Contendas, dans le Sertao des Minas, donné par Mr. A. Nogueira Duarte 15 [ex herb. A. St.-Hilaire, 1816-21]”, LourTEIG, 1986: 46.

$=$ C. viscosissima var. parietarioides A. St.-Hil., Fl. Bras. Merid. 3: 111. 1833. $\equiv$ C. parietarioides (A. St.-Hil.) Koehne in Engl. in Bot. Jahrb. Syst. 2: 170. 1881. Ind. loc.: «In pascuis prope vicum Contendas, parte occidentali desertàque provinciae Minas Geraes dictâ Sertao». Tipo en P: "Brasil, Minas Geraes, patürages près le village Contendas, A. Saint-Hilaire 630, 1816-21” (LourTEIG, 1986: 46), n. v.

=C. petiolata Pohl ex Koehne in Mart., Fl. Bras. 13(2): 230, 298-299, lám. 54, 5 . 1877, non C. petiolata (L.) Koehne in Bot. Jahrb. Syst. 2: 170. 1882. Ind. loc.: «Habitat ad Rio Buccaiuva prov. Minas Geraes: Pohl n. 2418 et 5732». Lectótipo en W!: "Brasil, Minas Geraes, Rio Boccaiuva, Pohl 2418" (designado por LouRTEIG, 1986: 47).

= C. costata Koehne in Mart., Fl. Bras. 13(2): 298, lám. 55, 1. 1877. Ind. loc.: «Habitat in provincia Minas Geraes: Pohl n. 5735; ad Rio S. Catharina: id. n. 3431; inter Fazendas Piccado et Cedro: id. n. 5730; ad Lagoa Santa: Warning». Lectótipo en C: "Brasil, Minas Geraes, Lagôa Santa, Warming s. n." (designado por LourTEIG, 1986: 47) n. v. Isolectótipos en G!, P!, S!, W!.

$=C$. chodatiana Koehne in Engl. in Bot. Jahrb. Syst. 2: 147. Ind. loc.: «In collibus pr. Tobaty, Sept. n. 6138». Holótipo en G!: "Prope Tobaty in collibus / September 1900 / E. Hassler 6138»; pliego que contiene un ejemplar incompleto a la izquierda y un fragmento de inflorescencia a la derecha.

= C. talaverensis Koehne in Feddes Repert. Spec. Nov. Reg. Veg. 8: 197-198. 1910. Ind. loc.: «Nördlicher Chaco: Puerto Talavera, September, Fiebrig nº 1226 (Herb. Hassler)». Holótipo en G!: "Chaco septentrionalis: Puerto Talavera / Sept. 1906 / Fiebrig: 1226” (LourTeIG, 1986: 47, como holótipo). Isótipos en G!, S!, Z!. 
= C. rojasi Koehne in Feddes Repert. Spec. Nov. Regni Veg. 8: 198-199. 1910. Ind. loc.: «Nord-Paraguay: Sierra de Amambay, auf sandigen Campos bei Esperanza. Februar. T. Rojas in Hassler. Pl. parag. $n^{\circ}$. 10756». Holótipo en G!: "Sierra de Amambay in campis arenosis / Esperanza / Febr. 1907-08 / T. Rojas s. n. / (ex herb. Hassler 10756)" (LourTeIG, 1986: 47). Isótipos en BM!, G!, P!, S!, W!.

$=C$. chiquitensis Herzog in Meded. Rijks Herb. Leiden 27: 16. 1915. Ind. loc.: «Im Kamp von Santiago de Chiquitos, ca. 600 m (n .510 der erten Reise, bl. im Mai 1907)». Holótipo en L 7254: "Flora von Bolivia / In kamp von Santiago de / Chiquitos, ca. $600 \mathrm{~m} / \mathrm{Mai}, 1907$ / leg. Th. Herzog 510" (LourTEIG, 1986: 47). Isótipo en $\mathrm{Z}$ !

$=$ C. trichopetala Rusby \& Koehne in Mem. Torrey Bot. Club 6(1): 40. 1896. Ind. loc.: «Vic. Cochabamba, 1891 (1008)». Holótipo en NY: «Bolivia, Bolivian Plateau, M. Bang 1008a, 1891» (LourteIG, 1986: 47), n. v. . Isótipos en BM!, M!, P!, US, W!, WIS.

- C. hispida Pohl ex Koehne in Mart., Fl. Bras. 13(2): 297. 1877, nom. nud.

Sect. Melvilla Koehne, Ind. Sem. Hort. Bot. Berol., app. 2, 1873.

Tipo: C. melvilla Lindl. (tipificación automática, Art. 22. 5 CINB).

= Melvilla Anderson ex Rafin., Sylva Tell.: 102. 1838 [- Melvilla Anderson

in Trans. Soc. London Encour. Arts 25: 207. 1807, nom. nud. ]. Tipo (designado aquí): Melvilla speciosa Anderson ex Rafin., 1. c.

= Sect. Balsamonae subsect. Pseudolobelia Koehne in Engl. in Bot. Jahrb. Syst. 2: 399. 1882.

23. Cuphea melvilla Lindl. in Bot. Reg. 10: 852-853, lám. 852. 1824.

Ind. loc.: "native of swamps in Guiana, and in Fort Zeland, Island of Essequeba whence it was introduced to the Botanic Garden, St. Vicent's, by the late Dr. Anderson". Lectótipo: [Icon] Lám. 852 in Lind., 1. c. (escogido por LouRTEIG, 1989: 28).

= Melvilla speciosa Anderson ex Rafin., Sylva Tell.: 102. 1838 [- Melvilla speciosa Anderson in Trans. Royal Soc. Arts, London 25: 207. 1807, nom. nud. ].

- Cuphea speciosa (Anderson) Kuntze, Revis. Gen. Pl. 3(2): 96. 1898, nom. inv.

24. Cuphea niederleinii Koehne in Bot. Jahrb. Syst. 15 (38): 5. 1893.

Ind. loc.: «Argentina, Misiones: Expedición de la comisión Argentina de límites: Picadas a Campinas de América, entre los arroyos Piedra Blanca, Bonito y Alemán, Yerbales y Picadas de Misiones, 2. nov. 1886: Niederlein n. 1036 (676)! Salto del Encuentro, Rio Piray, Misiones, 9. dec. 1886: Niederlein n. 1031». Material tipo: Según LourTeig (1943), el pliego Niederlein 1036 (=676) que se conservaba en B fue destruido por el fuego y se conserva la foto 17902 , que no hemos visto. También se conserva un duplicado en BA, que tampoco hemos visto. Lo más razonable parece lectotipificar sobre este material de BA.

Cuphea P. Br. subgen. Lythrocuphea Koehne, Ind. Sem. Hort. Bot. Berol., appendix altera. 1873. 
Tipo (designado aquí): C. racemosa (L. fil.) Spreng. [三Lythrum racemosum L. fil. ].

Sect. Enantiocuphea Koehne in Mart., Fl. Bras. 13(2): 241. 1877.

Tipo (designado aquí): C. punctulata Koehne.

25. Cuphea fruticosa Spreng. in Neue Entd. 2: 156. 1821.

Ind. loc: «in Brasilia». Material tipo en BM 512798: «Brazil, 1815-17, F. Sello».

$=C$. ligustrina Cham. \& Schltdl. in Linnaea 2: 359. 1827. Ind. loc.: «ad proedium Mandiocca in provincia Rio de Janeiro...». Material tipo: localización desconocida.

$=C$. ligustrina Cham. \& Schltdl. var. commersoniana A. St.-Hil., Fl. Bras. Merid. 3: 127. 1833. Ind. loc.: «Et à Montevideo près Ripis Ribularum circa Laumas aquarum dulibum». Holótipo en P!: "Montevideo, II-1767, Commerson" (LourTeIG, 1964: 121). $\equiv$ C. commersoniana (A. St.-Hil.) Koehne in Mart., Fl. Bras. 13(2): 241. 1877.

26. Cuphea punctulata Koehne in Mart., Fl. Bras. 13(2): 248, t. 43, f. 1. 1877.

Ind. loc.: «Habitat in udis in rirorum saxis provinciarum Bahía et Piauhy: Coulon, Blanchet n. 1635, Riedel n. 81, Martius». Lectótipo: (designado aquí) en M: «Habitat in urbis, ad rivas / Provinciae Piauhiensis / Dr. Martius Iter Brasil. Maio». Pliego conteniendo 2 ejemplares. El material de B destruido, hay que escoger entre el síntipo disponible.

27. Cuphea racemosa (L. f.) Spreng., Syst. Veg. 2: 455. 1825, s. str.

$\equiv$ Lythrum racemosum L. f., Suppl. pl.: 250. 1781. Ind. loc.: «Habitat in America meridionali. Mutis». Lectótipo en S: Mutis 72 [LINN 626-15!] (designado por LOURTEIG, 1989: 37).

$=$ C. spicata Cav., Icon. 4(2): 56, t. 381. 1797. Ind. loc.: «In provincia Canta Regni peruviani praesertim in monte vulgo de la Viuda iuxta aquas». Material tipo en MA (GARILleti, 1993: 156). Lectótipo (designado aquí) en MA-Cavanilles (!): «Peru, Prov. Canta, Monte de la Viuda, Née».

=C. obtusifolia Koehne ex Bacigalupi in Contr. Gray Herb. 95: 5-6, lám. I. 1931. Ind. loc.: «Brasil, Paraná, Piraguara on marshy ground». Lectótipo en S! (ex Herb. Regnell.): "Piraquara in paludosis, 7-1-1909, P. Dusén 7782" (LourTEIG, 1964: 122).

$=$ C. racemosa var. discolor Lourt. in Lilloa 9: 341-342, lám. 1. 1943. Ind. loc.: “Argentina, Misiones, Santa Ana, 16-II-1913, Rodríguez, s. n.". Holótipo en LIL 65120!: "Argentina, Misiones, Santa Ana, 16-II-1913, Rodríguez, s. n.».

Sin duda se trata de un taxon polimorfo que muestra gran variación en el hábito, indumento y en algunos caracteres florales y del fruto, sin que se conozca con certeza su correlación con factores ecológicos o la distribución geográfica. Para no perder información, hemos preferido un tratamiento analítico y jordaniano de este taxon, puesto que en un trabajo limitado territorialmente al Paraguay aún es posible distinguir estas categorías, a pesar de la opinión contraria de LOURTEIG (1964: 122). Una interpretación definitiva deberá basarse en un estudio más amplio en toda el área de distribución de la especie y en el cultivo experimental. En particular, las diferencias 
entre la var. racemosa y la var. palustris (anual versus perenne) pudieran deberse más a modificaciones morfológicas en la segunda por su condición de perennante, que a microcaracteres fijados como respuesta a factores ecológicos o de otro tipo. Al estudiar el material de herbario, se observan sutiles y constantes diferencias entre estos dos táxones que contradicen la idea de integrarlos como meros sinónimos, por más que aparezca algún pliego de difícil adscripción.

27a. C. racemosa var. palustris Lourt. in Lilloa 9: 342-344, lám. 2. 1943.

Ind. loc.: "Argentina, Chaco, Fontana». Holótipo en LIL: Meyer 3060 (LOURTEIG, 1943: 342), n. v.

Hemos consultado muy abundante material de herbario de este taxon, habitualmente identificado como $C$. origanifolia Cham. \& Schltdl. (in Linnaea 2: 373. 1827), sin duda el sinónimo más frecuente. De la amplia sinonimia disponible para este taxon (KoEHNE, 1903), conviene retener los trinómenes C. spicata var. tropica Cham. \& Schltdl. (1. c.) y C. spicata var. extratropica Cham. \& Schltdl., (l. c.), alguno de los cuales podría ser prioritario sobre la var. palustris si se confirma su homonimia. De momento, no hemos podido localizar material tipo de estos táxones.

27b. C. racemosa var. ramosior (Koehne) Duré \& Molero, comb. nov.

$\equiv$ C. origanifolia Cham. \& Schltdl. var. ramosior Koehne in Bot. Jahrb. Syst. 1: 450. 1881. Ind. loc.: "S. Paulo: Mug?". Material tipo no encontrado.

$=C$. hirticaulis Koehne in Bot. Jahrb. Syst. 41: 81-82. 1908. $\equiv$ C. racemosa subsp. hirticaulis (Koehne) Lourteig in Sellowia 16: 122-123. 1964. Ind. loc.: «Paraguay, Caaguazú». Holótipo en G: "E. Hassler, Plantae Paraguarienses / n 9205 / (unicum) / Prope Caaguazú in dumetis / Mart.".

$=$ C. correntina Lourteig in Darwiniana 8(2, 3): 266. 1948. Ind. loc.: "Corrientes, Dpto. Santo Tomé, Gobernador Vírasoro». Holótipo en LIL 116574: "Corrientes, Dpto. Santo Tomé / Gobernador Vírasoro / 11-XI-1944 / flor amarilla / T. S. Ibarrola” (LOURTEIG, 1. c.).

La var. ramosior es nombre prioritario a su nivel sobre $C$. hirticaulis. Aunque no hemos hallado material tipo de la var ramosior, establecemos la sinonimia de acuerdo con el criterio del propio Koehne, explicitado en las etiquetas de numerosos pliegos de herbario.

LOURTEIG (1948) diferenció C. correntina de los táxones próximos C. racemosa, $C$ hirticaulis y $C$. longiflora esencialmente por el color de las flores, que especifica amarillo atendiendo a la indicación de la etiqueta del material tipo, aunque ciertamente el colector Ibarrola antepuso primero la palabra «azul-rosada», que luego tachó y añadió: «flor amarilla». Realmente la descripción del protólogo de C. correntina es muy próxima, si no idéntica, a la var. hirticaulis, exceptuando el color de la flor. Un examen comparativo del material tipo de $C$. correntina y de algún otro material del Paraguay con el tipo de $C$. hirticaulis no muestra ninguna diferencia importante, tal vez el indumento de los tallos, algo menos denso. El color de las flores en el complejo $C$. racemosa puede ser algo variable, presentándose raramente flores albinas tanto en la var. hirticaulis como en la subsp. longiflora. En material seco de herbario estas flores albinas pueden aparecer como amarillentas. Creemos que éste es el caso 
de la especie que nos ocupa. Las razas albinas no merecen otra consideración que el de forma. Posiblemente Lourteig se dejó sugestionar por este carácter tan llamativo. Realmente los materiales observados de la var. hirticaulis, y especialmente los atribuibles a $C$. correntina, presentan un hábito, hojas, inflorescencia y tamaño de las flores muy próximo a la subsp. longiflora, aunque difieren en el tipo de indumento y su distribución en tallos y hojas; también las semillas son anchamente marginadas, casi aladas, carácter más típico de la subsp. longiflora. De hecho se trata de formas de transición que justificarían el tratamiento de $C$. longiflora como subespecie.

27 c. Cuphea racemosa subsp. longiflora (Koehne) Duré \& Molero, comb. nov.

$\equiv$ C. longiflora Koehne in Mart., Fl. Bras. 13(2): 246, t. 42, f. 2. 1877.

Ind. loc.: «Habitat in umbrosis paludibusque provinciarum Mines Geraes et S. Paulo». Lectótipo (designado aquí): [Icon] Cuphea longiflora Koehne, Fl. Bras. 13(2): 246, t. 42 , f. 2.1877.

$=$ C. longiflora forma major Koehne in Mart., Fl. Bras. 13(2): 247. 1877. Ind. loc.: “Widgren n. 478; Lindberg n. 348; ad margenem Rio Grande: Lund; Lorena: Riedel". Material tipo no localizado, posiblemente destruido.

$=$ C. longiflora forma minor Koehne in Mart., Fl. Bras. 13(2): 247. 1877. Ind. loc.: Lindberg 349. Material tipo: como el anterior.

28. Cuphea bonplandii Lourteig in Lilloa 9: 346-348. 1943.

Ind. loc.: «Argentina, Corrientes, Paso de la Patria». Holótipo en LIL: Meyer 2159 (LOURTEIG, l. c.), n. v.

= C. origanifolia var. gracillima A. St.-Hil. in Fl. Bras. Merid. 3: 102-103. 1833, syn. nov. Ind. loc.: «In Brasilae prope civitatem S. Pauli». Material tipo en P: Riedel 1592.

Las poblaciones del Paraguay, de ecología claramente palustre, presentan un porte más grácil, ascendente, con hojas y flores más pequeñas que las poblaciones argentinas, más robustas y de porte suberecto; el material paraguayo se asemeja más al material de herbario estudiado procedente del Brasil (etiquetados como $C$. origanifolia var. gracillima). No obstante, los caracteres florales, el indumento y las semillas se mantienen constantes en toda el área de distribución.

\section{AGRADECIMIENTOS}

Los autores desean expresar su agradecimiento a la Dra. Alicia Lourteig, que atendió con gran amabilidad las consultas de R. Duré, durante su estancia, en 1998, en el Museo de Historia Natural de París. Igualmente desean agradecer las gestiones puntuales del Dr. E. Vitek en el herbario W y del Dr. Ch. Jarvis en el herbario BM respecto a la búsqueda de material tipo de Lythrum racemosum. También debemos agradecimiento a L. Ramella y P. Perret, del Conservatoire Botanique de Genève, por su apoyo bibliográfico y de herbario. Este trabajo se ha beneficiado de una ayuda a proyectos de investigación conjuntos para el trienio 1998-2000 del Programa de Cooperación Científica con Iberoamérica (MEC-AECI) del gobierno español. 


\section{REFERENCIAS BIBLIOGRÁFICAS}

D'ARCY, W. G. (1970). Jacquin names, some notes on their typification. Taxon 19: 554-560.

DurÉ, R. (1999). Revisión del género Cuphea P. Br. (Lythraceae) en el Paraguay. Tesis Doctoral Universidad de Barcelona. Barcelona.

Garilleti, R. (1993). Herbarium cavanillesianum. Fontqueria 38: 1-248

Graham, S. A. (1990). New species of Cuphea Section Melvilla (Lythraceae) and an annotated key to the Section. Brittonia 42(1): 12-32.

Graham, S. A. (1998). Relacionamientos entre as espécies autógamas de Cuphea P. Brownw seçao Brachyandra Koehne (Lythraceae). Acta Bot. Bras. 12(3): 203-214.

Hassler, E. (1898). Lythraceae. In Chodat \& Hassler (ed.). Plantae Hasslerianae. Bull. Herb. Boissier 6(1): 72-73.

Hassler, E. (1902). Lythracées. In Chodat \& Hassler (ed.), Plantae Hasslerianae. Bull. Herb. Boissier 2a. sér. 2: 401-402.

Hassler, E. (1909). Florula Pilcomayensis. 154 pp. Buenos Aires.

KoeHNe, (1903). Lythraceae. In Engler, Pflanzenreich 17 (IV. 216): 1-326; figs. 1-59. Leipzig.

KoEHNE, E. (1908). Lythraceae monographie describentur. In Engler in Bot. Jahrb. Syst. 41: 74-110.

Koenne, E. (1910 a). Neue Lythraceae aus Paraguay und dem Gran Chaco I. Feddes Repert. Sp. Nov. Regni Veg. 8: 165-167.

Koenne, E. (1910 b). Ex Herbario Hassleriano. Neue Lythraceae aus Paraguay und dem Gran Chaco II. Feddes Repert. Sp. Nov. Regni Veg. 8: 196-199.

LourTeig, A. (1943). Lythraceae Argentinae. Lilloa 9: 317-421.

Lourteig, A. (1948). Lythraceae Argentinae. Addenda II. Darwiniana 8(2-3): 263- 278.

Lourteig, A. (1964). Lythraceae Austroamericanae. Addenda et Corrigenda. Sellowia 16: 119162.

LourTeIG, A. (1969). Litráceas. In P. R. Reitz (ed.), Flora Ilustrada Catarinense. 80 pp. Itajaí, Santa Catarina, Brasil.

Lourteig, A. (1986). Revisión de dos secciones de género Cuphea P. Browne (Lythraceae). Phytologia 60(1): 17-55.

LourTeIG, A. (1987). Lythraceae austroamericanae. Addenda et Corrigenda. II. Sellowia 39: $5-48$.

Lourteig, A. (1989). Lythraceae. En G. Harling \& L. Anderson (eds.). Flora of Ecuador 37: 147.

Molero, J. \& G. Zulsstra (1999). The typification of Cuphea (Lythraceae): C. viscosissima versus $C$. decandra. Taxon 48(4): 797-799. 